\title{
Ein Kampf gegen Windmühlen? Zur Prävention von Alltagsdelikten
}

\author{
Susanne Knickmeier
}

\section{$1 \quad$ Einleitung}

Im April 2017 berichtete der deutsche Zoll, dass im Jahr 2016 durch festgestellte Schwarzarbeit und illegale Beschäftigung eine Gesamtschadenssumme in Höhe von 876 Millionen Euro entstanden sei (vgl. Bundesministerium der Finanzen 2017, S. 18). Der Verband Deutscher Verkehrsunternehmen (VDV) gab im Jahr 2015 in einer Pressemitteilung an, dass deutschen Verkehrsbetrieben jährlich ein Schaden von 250 Millionen Euro durch Schwarzfahren entstehe (vgl. VDV 2015). Bei Kontrollen zur Produktpiraterie beschlagnahmte der deutsche Zoll im Jahr 2016 3,46 Millionen gefälschter Waren im Wert von 180 Millionen Euro (vgl. Bundesministerium der Finanzen 2017, S. 12). Aus Urlaubsländern werden gerne günstige Waren mitgebracht, zum Beispiel Zigaretten, technische Geräte und nachgeahmte Kleidung, die die erlaubten Reisefreimengen überschreiten oder nicht zum Eigenbedarf bestimmt sind und für die keine Zollabgaben geleistet werden. Der Download von Fernsehserien, Computerspielen oder Musik erfreut sich großer Beliebtheit. Nicht jeder ${ }^{1}$ zahlt gerne für die Nutzung kostenpflichtiger Daten, sondern lädt sie sich unter Verstoß gegen Urheberrechte aus dem Internet. Täglich sind in Deutschland Tausende von den sogenannten Alltagsdelikten ${ }^{2}$ betroffen, sei es als Arbeitgeber von Personen, für die kei-

1 Aus Gründen der besseren Lesbarkeit wird auf die gleichzeitige Verwendung männlicher und weiblicher Sprachformen verzichtet. Sämtliche Personenbezeichnungen gelten gleichwohl für beiderlei Geschlecht.

2 Der Begriff des Alltagsdelikts ist nicht einheitlich definiert. Im vorliegenden Beitrag werden darunter die Delikte verstanden, mit denen die Polizei täglich konfrontiert ist, bei denen der Schaden gering und die Strafandrohung nicht hoch ist. Alltagsdelikte können zum Beispiel sein: Fahren ohne Fahrausweis, der grenzüberschreitende Transport von Alkohol oder Zigaretten, ohne die Beachtung von Reisefreimengen, der Kauf gefälschter Waren, das illegale Herunterladen von Musik, Spielen, Filmen, die Beschäftigung von Schwarzarbeitern. 
ne Sozialversicherungsabgaben geleistet werden, Schwarzarbeiter, als Fahrgast ohne gültigen Fahrausweis, Händler, Schmuggler oder Käufer unerlaubter Waren oder als Opfer (zum Beispiel als Hersteller von Markenkleidung, Steuerzahler oder unwissender Käufer gefälschter Produkte). Der unerlaubte Markt oder das Vorenthalten von Fahrpreisen, Sozialversicherungsbeiträgen und Steuern verursacht große Schäden für die nationale Wirtschaft, den legalen Markt, den Arbeitsmarkt, die Steuer- und Zolleinnahmen und unter Umständen sogar die Gesundheit. Auch wenn das einzelne Delikt unterhalb von Bagatellgrenzen liegen kann, ist der volkswirtschaftliche Schaden hoch (zum Hellfeld: siehe oben). Schätzungen zufolge haben im Jahr 2016 rund drei Millionen Haushaltshilfen in Privathaushalten unerlaubt gearbeitet, was einem geschätzten Schaden von 140 Milliarden Euro entspricht (vgl. Enste 2017, S. 3). Der Bundesverband Musikindustrie (BMVI) vermutet, dass durch das illegale Herunterladen von Musikaufnahmen in Deutschland jährlich ein Schaden in dreistelliger Millionenhöhe bei Songschreibern, Künstlern, Musikindustrie und dem steuereinnehmenden Staat verursacht wird (vgl. BVMI o. D.)

Die angezeigten oder entdeckten Delikte sind nur die Spitze eines Eisberges, bei dem von einer hohen Dunkelziffer auszugehen ist. Von den begangenen Straftaten wird ein Großteil der Taten nicht entdeckt, nicht angezeigt oder wenn sie angezeigt werden, nicht aufgeklärt. Alltagsdelikte werden auch von Personen begangen, die sich als angesehene Mitglieder der Gesellschaft verstehen und nach Karstedt und Farrall die Bezeichnungen „Straftat" und "kriminell“" weit von sich weisen würden (vgl. Karstedt und Farrall 2006, S. 1011). Gerade im Hinblick auf sie ist die Frage, mit welchen Maßnahmen Alltagsdelikten effektiv begegnet werden kann, kriminalpolitisch besonders interessant. Gängige kriminalpolitische Maßnahmen zur Erreichung von Normkonformität sind formelle und informelle Kontrollen zur Entdeckung und anschließenden Sanktionierung abweichenden Verhaltens. Für Strafverfolgungsbehörden ist es ein Problem, der großen Anzahl von Alltagsdelikten ${ }^{3}$, die erhebliche personelle und finanzielle Ressourcen verbrauchen, Herr zu werden. Der Landesvorsitzende der deutschen Polizeigewerkschaft Nordrhein-Westfalen schlug als Maßnahme bei Alltagsdelikten vor, niederschwellige Delikte als Ordnungswidrigkeit zu verfolgen, damit die Polizei ihre Ressourcen auf eine effektive Bekämpfung schwerer Straftaten fokussieren kann (vgl. Gewerkschaft der Polizei 2015). Fraglich ist, ob Kontrollen, Strafen oder die Verfolgung von Alltagsdelikten als Ordnungswidrigkeit effektiv sind oder aufgrund der hohen Anzahl von Delikten auch normative Maßnahmen in Erwägung zu ziehen wären, um Normkonformität durch institutionelle Legitimität und Vertrauen in das Rechtssystem zu erreichen.

3 Der vorliegende Beitrag behandelt keine präventiven Maßnahmen zur Bekämpfung der organisierten Kriminalität, sondern bezieht sich auf die Personen, die Waren in kleinem Rahmen unerlaubt handeln, auch wenn sie von Personen, die der organisierten Kriminalität zuzurechnen sind, verkauft werden. 


\section{Kriminalpolitische Ansätze}

In demokratischen Gesellschaften wird das soziale, wirtschaftliche und politische Leben anhand von Gesetzen, Verordnungen oder Richtlinien reguliert, an die sich der einzelne Bürger halten soll. Auch wenn einige Personen gegen rechtliche und soziale Normen verstoßen, werden sie von einem Großteil der Bevölkerung befolgt, so dass sich die Frage stellt, welche Maßnahmen rechtstreues Verhalten fördern. Dazu werden vor allem zwei Ansätze diskutiert, die sich ergänzen können und nicht ausschließen müssen: (1.) repressive Strategien, wie vermehrte Kontrollen und verschärfte Gesetze (sog. instrumental compliance) und (2.) normative Strategien, die die Überzeugung von der Richtigkeit und Legitimität einer Norm beinhalten (sog. normative compliance) (vgl. Bottoms 2001, S. 90).

\subsection{Repressive Strategien}

Repressive Strategien gehen davon aus, dass ein potentieller Täter normkonformes Verhalten als sein eigenes Interesse ansieht, weil er eine Bestrafung vermeiden möchte (vgl. Bottoms 2001, S. 9o f.). Die repressiven Maßnahmen beruhen oft auf der Rational-Choice-Theorie, die das Leitbild des neoklassischen Homo Oeconomicus voraussetzt. Im Idealtypus ist der Homo Oeconomicus ein ausschließlich wirtschaftlich denkender Mensch mit einem uneingeschränkt rationalen Verhalten und dem Streben nach Nutzen- oder Gewinnmaximierung (vgl. Lin-Hi o. D.). Nach der RationalChoice-Theorie kann ein potentieller Straftäter durch externe Anreize abgeschreckt werden, wenn die Kosten für die Begehung der Straftaten (Strafverfolgungsrisiko, Strafschwere) höher sind als der materielle Vorteil (vgl. Becker 1968, S. 207f.). Zur Herstellung von Normkonformität stützen sich repressive Strategien daher vor allem auf verstärkte Kontrollmaßnahmen, um das Entdeckungsrisiko zu erhöhen, sowie auf die Verschärfung von (Straf-)Gesetzten. Für den Fall eines Gesetzesverstoßes wird eine Strafe angedroht, die bei einem Verstoß verhängt und vollstreckt wird. Zur Erhöhung des Entdeckungsrisikos werden z.B. (Grenz-)Kontrollen, auch technisch, verstärkt oder der Austausch von Daten zwischen Strafverfolgungsbehörden gefördert. Auch Wirtschaftsunternehmen investieren, soweit sie von Straftaten, wie zum Beispiel der Produktpiraterie, betroffen sind, viel in die (technische) Entwicklung von Präventionsmaßnahmen, gründen Kooperations-Netzwerke, kooperieren mit Strafverfolgungsbehörden (zum Beispiel bei Datenbanken über Produktpiraterie), entwickeln technische Maßnahmen (zum Beispiel das Track- und Trace-System, mit dem Zigaretten von ihrer Produktion bis zum Verkauf verfolgt werden können) oder führen, wie Verkehrsunternehmen, selber Kontrollen durch.

Auch wenn das Leitbild des Homo Oeconomicus bis heute in der Gesellschaft vorherrscht, stellt sich die Frage, ob Menschen wirklich so rational handeln, denken und entscheiden. Welche Motivationssysteme bestimmen das menschliche Handeln? 
Wird die menschliche Entscheidung nicht auch durch interne Anreize beeinflusst, indem die Befolgung von Normen als Vorteil und lohnenswertes Handlungs- oder Entscheidungsmotiv wahrgenommen wird? Beeinflusst nicht auch der Wunsch nach einer positiven Gestaltung des Umfeldes, einem kooperativen und fairen Verfahren sowie Vertrauen das Handeln? Reagiert der Mensch nicht auch auf intrinsische Anreize, wenn für ihn die Einhaltungen von Regeln und Normen eine lohnenswerte Handlung ist? Die Neurowissenschaftlerin Singer kritisiert, dass der Homo Oeconomicus die Komplexität des Menschen und seiner Entscheidungsfindung nicht angemessen wiedergebe (vgl. Reich 2015). Neurowissenschaftliche Studien haben gezeigt, dass sich Menschen ebenso durch Fürsorge und Zugehörigkeitssysteme motivieren ließen (vgl. Singer 2015). Falk, der das Modell des Homo Oeconomicus empirisch untersuchte, betont, dass bei Entscheidungen auch Fairness und Reziprozität eine Rolle spielen; ein Verhalten, das dem Homo Oeconomicus fremd ist (vgl. Falk 2001, S. 2 ff.).

\subsection{Normative Strategien}

Normative Erwägungen, die das Vertrauen der Bürger in die Legitimität des (Straf-) Rechts sowie die Legitimität des nationalen Justizsystems fördern, aber auch den potentiellen Täter bzw. Abnehmer einer unerlaubten Leistung von der Richtigkeit der Norm überzeugen wollen, spielen bei der Kriminalprävention bislang eine untergeordnete Rolle. Nach Tyler lassen sich potentielle Straftäter aber nicht nur von rationalen Faktoren leiten, sondern sie beziehen normative Erwägungen ebenfalls ein:

„Wenn Menschen die Einhaltung von Rechtsnormen als angemessen betrachten, weil die Normen mit ihren Einstellungen, wie man sich zu verhalten habe, übereinstimmen, werden sie freiwillig die Verpflichtung, sich an diese Rechtsnormen zu halten, übernehmen “4 (Tyler 1990, 3).

Dem Ansatz normativer Strategien folgend verhalten sich Personen dann normkonform, wenn sie von einer Norm überzeugt sind, ihre Legitimität akzeptieren und davon ausgehen, das moralisch Richtige zu tun (vgl. Bottoms 2001, S. 91). Normative Strategien zielen darauf, dass ein potentieller Täter nicht länger den Willen hat, eine Straftat zu begehen, weil er aus moralischen Gründen dagegen ist. Für ihn besteht also eine Kongruenz von Recht und moralischer Einstellung. Selbst wenn die moralischen Vorstellungen des Einzelnen nicht mit allen existierenden Regeln übereinstimmen, was in einer demokratischen Gesellschaft, in der die Normen mehrheitlichen Entscheidungen unterliegen, üblich ist, erkennt er an, dass es rechtlicher Regelungen 
bedarf, die zur Aufrechterhaltung der sozialen Ordnung einzuhalten sind. Er hält es für richtig, sich an das Recht zu halten, selbst wenn es nicht zum eigenen Vorteil ist (vgl. Jackson et al. 2012, S. 1053). Die Entscheidung, sich aus moralischen Gründen zu rechtstreuem Verhalten verpflichtet zu fühlen, kann auch mit dem internen Anreiz des guten Gefühls, sich wie ein ehrlicher Mensch verhalten zu haben, verbunden sein. Nach Mazar und Ariely können die Selbstwahrnehmung als anständig oder ehrlich und die Übereinstimmung zwischen Selbstbild und Handeln entscheidungsrelevant sein (vgl. Mazar und Ariely 2006, S. 119 ff.). Die Förderung solcher interner Anreize kann besonders bei potentiellen Tätern im Bereich der Alltagskriminalität, die sich für anständige Mitglieder der Gesellschaft halten, wichtig sein.

\subsubsection{Procedural Justice Theory}

Die zur Regulierung sozialen Verhaltens entwickelten normativen Erwägungen umfassen nach der von Tyler geprägten, empirisch untersuchten Procedural Justice Theory (1.) das Vertrauen in die Legitimität von Recht, (2.) das Vertrauen in die Legitimität von Institutionen und (3.) die Verfahrensgerechtigkeit. Wenn Menschen davon ausgehen, dass die Staatsmacht und ihre Gesetze legitim sind und sie justiziellen Institutionen wie Polizei und Gerichten vertrauen, sind sie bezugnehmend auf Tyler geneigter, geltende Normen anzuwenden und mit dem Justizsystem zu kooperieren (vgl. Tyler 2003, S. 284). Der Begriff der Legitimität an sich bezieht sich auf die Anerkennung des Staates, seiner Rechtmäßigkeit, seiner Herrschaftsakte sowie die Einhaltung von ethisch-normativen Vorstellungen der Allgemeinheit und nicht nur der Herrschenden (vgl. Beetham 1991, S. 71; Glaser 2013, S. 15). In einem als legitim anerkannten Staat beruhen vom Gesetzgeber erlassene rechtliche Normen meistens auf den mehrheitlichen, moralischen Einstellungen der Gesellschaft (vgl. Hilgendorf 2001, S. 75, 78). Wann aber werden Institutionen als legitim perzipiert? Tyler geht mit der Procedural Justice Theory davon aus, dass Institutionen dann als legitim wahrgenommen werden, wenn das Verfahren an sich und nicht der Verfahrensausgang als gerecht angesehen wird (vgl. Tyler 2003, S. 284).

Vertrauen in die Legitimität von Polizei, Gerichten und anderen justiziellen Institutionen kann durch folgende Modi Procedendi gefördert werden: (1.) Verfahrensgerechtigkeit, (2.) Verteilungsgerechtigkeit und einem gerechten Verfahrensausgang, (3.) eine bewiesene moralische Anpassung und (4.) durch bewiesene Kompetenzen der handelnden Institution. Für die Herstellung von Vertrauen in die Legitimität des Strafrechts sind wichtig: (1.) Anerkennung der Legitimität von justiziellen Institutionen, (2.) eine Kongruenz von Recht und Moral und (3.) die Entfernung moralisch tolerierten Verhaltens aus dem Regelungsbereich des Strafrechts. Sich innerhalb der Gesellschaft ändernde moralische Vorstellungen führen meist (zeitversetzt) zu einer Weiterentwicklung rechtlicher Normen. Dafür gibt es diverse Beispiele, unter anderem die Abschaffung des $\$ 175$ StGB (Strafbarkeit homosexueller Handlungen) im Jahr 1994. 


\subsubsection{Gruppenidentfikation}

Zur Beantwortung der Frage, wie die Kongruenz von Recht und Moral sowie Anerkennung der Legitimität von Institutionen verbessert werden kann, wird auf sozialpsychologische Mechanismen der Identifikation mit Gruppen und Internalisierung von Normen zurückgegriffen. Institutionen, die Normen und Werte umsetzen, wirken auf den Einzelnen mit identitäts- und statusrelevanten Informationen und führen zur Entwicklung einer persönlichen Identität unter Einbeziehung von Erfahrungen und Wissen (vgl. Jackson et al. 2012, S. 1053; Lind und Tyler 1988, S. 231 ff.). Zudem wird das Zugehörigkeitsgefühl zur Gesellschaft, die durch Persönlichkeiten, Normen und Werte symbolisch vertreten wird, gestärkt. Erfahrung von Ungerechtigkeiten, nicht respektvollem Verhalten und ungerechten Entscheidungen wiederum schwächen das Zugehörigkeitsgefühl (vgl. Jackson et al. 2012, S. 1053). Zur Erklärung, wie Verfahrensgerechtigkeit die Normkonformität des in der Gesellschaft integrierten Einzelnen beeinflussen kann, ziehen Lind und Tyler das Group Value Modell heran, das auf der sozialpsychologischen Theorie der sozialen Identität beruht (Tajfel und Turner 1986). Dem Modell folgend gehen sie davon aus, dass sich in einem Verfahren die Werte einer Gruppe oder Gesellschaft widerspiegeln, die dem Betroffenen wichtiger sind als der Ausgang des Verfahrens (vgl. Lind und Tyler 1988, S. 230 ff.; Tyler 1990, S. 173). Sind Personen in der Gesellschaft sowie ihren rechtlichen, politischen und sozialen Strukturen integriert und an sozialen Aktivitäten beteiligt, identifizieren sie sich mit der Gesellschaft, die wiederum Einfluss auf individuelle Einstellungen und Verhaltensweisen hat (vgl. Tyler 1990, S. 173). Gerade bei Tätern von Alltagsdelikten spielen soziale Akzeptanz und damit verbundene Gruppendynamiken eine besondere Rolle.

\subsection{Normkenntnis}

Trotz des Grundsatzes Ignorantia legis non excusat ist die Kenntnis, dass die vorgenommene Handlung strafbar ist, eine wichtige Voraussetzung für die erfolgreiche Umsetzung normativer Strategien. Vor allem bei Verstößen gegen die Einfuhr von Waren scheint es fraglich, ob die Täter wissen, dass für die von ihnen eingeführte Warenmenge Abgaben zu leisten sind. Während in die Schweiz fünf Liter alkoholischer Getränke mit bis zu 18 \% Alkohol eingeführt werden dürfen, beträgt die Menge vom EU-Ausland in die Europäische Union (EU) zwei Liter mit bis zu 22 \% Alkohol (vgl. Eidgenössische Zollverwaltung 2017; Zoll 2017b). Innerhalb der EU ist unter anderem zwischen Bier (110 Liter) und Spirituosen (zehn Liter) zu unterscheiden (vgl. Zoll 2017a). Fehlt es zudem an einer Verbindung von Recht, Rechtsempfinden und moralischem Grundkonsens über die Strafwürdigkeit eines Verhaltens, kommen potentielle Täter, die sich eigentlich normkonform verhalten wollen, gar nicht auf die Idee, die geltende Rechtslage zu prüfen. Ein weiterer Grund für eine fehlende Normkenntnis kann die Vielzahl rechtlicher Regelungen sein. Karstedt und Farrall (2006) beto- 
nen in dem Zusammenhang, dass zu viele Gesetze und Verordnungen die Anerkennung der Legitimität von Normen und moralischen Verpflichtungen senken und das illegale Verhalten mit der Folge normalisiert werde, dass es als richtig empfunden wird (vgl. Karstedt und Farrall 2006, S. 1017). Fehlendes Rechtsbewusstsein kann durch die Unkenntnis über den Entstehungsgrund der Norm oder eine fehlende Sensibilisierung über mögliche Verknüpfungen zur organisierten Kriminalität, Arbeitsausbeutung oder Kinderarbeit im Herstellungsland begründet sein (zum Beispiel bei geschmuggelten Zigaretten oder gefälschten Produkten). Bezugnehmend auf eine Online-Umfrage in Deutschland haben im Jahr 2009 nur die Hälfte der 454 Befragten gefälschte Produkte mit einer Gefährdung der Gesundheit verbunden (vgl. Vollmuth 2009). Niemand stellte in einer Meta-Analyse zur Produktpiraterie fest, dass Menschen vom Kauf von Raubkopien oder gefälschten Produkten abgesehen haben, wenn sie sich aus moralischen Gründen verpflichtet fühlten, ihr Verhalten zu unterlassen (Niemand 2014, S. 192). Der erste Schritt eines umfassenden kriminalpräventiven Konzepts sollte die Vermittlung von Normen, unbeabsichtigten Folgen unerlaubten Verhaltens und ggf. die Überprüfung des geltenden Rechts im Hinblick auf einen tragfähigen Konsens in der Gesellschaft sein.

\section{Motive und Rechtfertigungsstrategien der Täter}

Bei der Suche nach effektiven Strategien zur Erhöhung der Normkonformität und Akzeptanz der Legitimität stellt sich auch die Frage, was die Täter zu ihrem Handeln motiviert und wie ihre Rechtfertigungsstrategien erklärt werden können.

\subsection{Motive}

Folgende Gründe kommen als treibende Faktoren oder Motive bei der Begehung von Alltagsdelikten in Betracht: (a.) ein zu hoher Preis des Originalprodukts oder der Dienstleistung (zum Beispiel bei der häuslichen Pflege), (b.) wirtschaftliche Benachteiligung, (c.) fehlende finanzielle Möglichkeiten zur Teilnahme am sozialen Leben, (d.) der Wunsch ein bestimmtes Produkt zu besitzen, ohne das Geld dafür ausgeben zu wollen oder zu können, (e.) fehlendes Unrechtsbewusstsein, (f.) die soziale Akzep$\tan z$ des abweichenden Verhaltens, (g.) das Gefühl durch die staatliche Gewalt unfair behandelt zu werden (zum Beispiel beim Nicht-Verzollen von Zigaretten aufgrund der als zu hoch empfundenen Steuern oder auch bei Schwarzarbeit) oder (h.) die teilweise unterlassene Verfolgung von unerlaubten Handlungen durch Strafverfolgungsbehörden ${ }^{5}$ (vgl. dazu die Untersuchung von Antonopolus 2008; Bräuninger und Stiller 2010; Jian und Cova 2012; Stead et al. 2013, Wagner 2011; Wilcox 2009).

5 Teilweise wird der Verkauf illegaler Zigaretten an Straßenständen geduldet, unter anderem weil eine 


\subsection{Rechtfertigungsstrategien}

Die Mehrheit der Täter stimmt mit den in der Gesellschaft existierenden rechtlichen Vorschriften größtenteils überein, verhält sich aber trotzdem teilweise konträr zu ihren Überzeugungen. Womit rechtfertigen Alltagskriminelle ihr Verhalten? Wie beschrieben, sehen sie sich als respektable Mitglieder der Gesellschaft und nicht als Kriminelle. Sie haben die rechtlichen und sozialen Regeln internalisiert ${ }^{6}$ und offensichtlich Techniken entwickelt, um ihr kriminelles Verhalten zu rechtfertigen.

\subsubsection{Neutralisierungstechniken}

Eine These zur Rechtfertigung abweichenden Verhaltens haben Sykes und Matza (1957) mit der Neutralisierungsthese entwickelt, die von einer Internalisierung der geltenden sozialen und rechtlichen Normen ausgeht. Die Täter akzeptieren auf der einen Seite die Normen, verstoßen aber trotzdem gegen sie. Ihr abweichendes Verhalten rechtfertigen sie anhand von Neutralisierungstechniken. Sykes und Matza beschreiben fünf Techniken, das eigene Verhalten zu neutralisieren: (1.) Verneinung der Verantwortung, (2.) Verneinung des Unrechts, (3.) Nichtanerkennung des Opfers, (4.) „Verdammung der Verdammenden“ und (5.) Berufung auf höhere Instanzen (vgl. Sykes und Matza 1957, S. 667 ff.). Vor allem die Technik "Verneinung des Unrechts“ spielt eine wichtige Rolle in Fällen von Alltagskriminalität, zum Beispiel beim Kauf illegaler Produkte oder Fahren ohne gültigen Fahrausweis. Die Täter verstoßen bewusst gegen das Recht, davon ausgehend, niemandem einen großen Schaden zuzufügen, und bewerten ihr abweichendes Verhalten als weniger falsch. In Herkunftsländern mit einer schwächeren Wirtschaft können Täter ihre Handlungen auch damit rechtfertigen, dass sie den illegalen Job brauchen, um ihr Überleben zu sichern (Berufung auf höhere Instanzen). Die Nichtanerkennung des Opfers ist gegeben, wenn Täter damit argumentieren, dass der Staat oder ein Unternehmen, das Produkte zu Höchstpreisen verkauft, dem Bürger das Geld aus der Tasche ziehe und sie mit ihrem Verhalten gegen die mächtigen Großen „kämpfen“.

\subsubsection{Moral Economy}

Bezugnehmend auf Thompson (1971) und seinen Ansatz einer Moral Economy kann abweichendes Verhalten von Personen, vor allem denjenigen, die nicht aus wohlhabenden Verhältnissen stammen, ein Widerstand gegen Ungleichgewichte im Markt

strafrechtliche Verfolgung wenig erfolgsversprechend ist. Vietnamesische Verkäufer in Berlin, zum Beispiel, führen nur wenige Stangen Zigaretten mit sich, was keine hohe Haftstrafe begründen würde, geben bei Kontrollen falsche Personalien an und verlassen umgehend Deutschland, um durch ein weiteres Mitglied der Schmugglergemeinschaft ersetzt zu werden (vgl. Hansen 2012).

6 Eine Ausnahme von dieser Gruppe sind die Personen, die sich normkonform verhalten wollen, aber - wie in Abschnitt 2.3 beschrieben - in Unkenntnis einer Vorschrift gegen sie verstoßen. 
sein. Die Menschen wollen am sozialen Leben teilhaben, fühlen sich durch den Ausschluss aufgrund mangelnder finanzieller Möglichkeiten unfair behandelt und stellen daher die Legitimität des Marktes sowie seine traditionellen Rechte und Praktiken in Frage (vgl. Thompson 1971, S. 94 ff.). Ein ähnliches Phänomen des Widerstandes kann beobachtet werden, wenn sich Menschen durch staatliche Gewalt, zum Beispiel die Polizei, ungerecht behandelt fühlen. Im Bereich der Alltagsdelikte können ein zu hoher Preis des Originalproduktes, hohe Steuern oder ein unausgewogenes PreisLeistungsverhältnis verbunden mit dem Wunsch ein bestimmtes Produkt kaufen zu können, dazu führen, Waren auf dem illegalen Markt zu beziehen oder für ein Produkt oder eine Dienstleistung nicht zu zahlen.

\section{Normatives Modell zur Erreichung von Normkonformität}

In den vorangegangenen Abschnitten wurde beschrieben, dass verschiedene Strategien zur Normkonformität führen können: (1.) Kontrollen und das Risiko einer Bestrafung, (2.) persönliche Moralvorstellungen oder (3.) die Anerkennung der Legitimität des Rechts, die auf der Verpflichtung zu gehorchen und der moralischen Übereinstimmung mit der Polizei beruht.

\subsection{Projekt Fiducia}

Fraglich ist, inwieweit diese theoretischen Überlegungen auf die Gesellschaft anwendbar sind. Zur Prüfung der genannten Fragen wurden in dem von der EU geförderten Projekt Fiducia: New European Crimes and Trust-based Policy ${ }^{7}$ Daten durch eine repräsentativen Befragung erhoben. ${ }^{8}$ Die Befragung wurde in sechs Ländern der EU (Bulgarien, Deutschland, England, Finnland, Italien, Litauen) sowie der Türkei mit einem standardisierten Fragebogen durch Telefon- oder Face-to-Face-Interviews durchgeführt. Im vorliegenden Beitrag werden nur die Ergebnisse der Befragung in Deutschland einbezogen. ${ }^{9}$ In Deutschland wurden insgesamt 769 Telefoninterviews (24\% Mobiltelefon und $76 \%$ Festnetz) mit Personen, die mindestens 16 Jahre alt waren, geführt.

7 Das Projekt FIDUCIA war ein von 2012-2015 durch das 7th Framework Programme for Research von der Europäischen Union gefördertes Kooperationsprojekt von wissenschaftlichen Instituten aus 13 Ländern. Weitere Informationen zu dem Projekt finden sich unter: http://www.fiduciaproject.eu.

8 An der Entwicklung des normativen Modells und des Fragebogens zur Durchführung der repräsentativen Befragung haben diverse Wissenschaftler aus den teilnehmenden Ländern mitgewirkt. Besonders zu danken ist Prof. Dr. Ben Bradford (University College London), Prof. Dr. Jonathan Jackson (London School of Economics) und Prof. Dr. Mike Hough (Birkbeck College London) für ihre wertvollen Arbeiten bei der Entwicklung des normativen Modells und der Analyse der erhobenen Daten, deren Ergebnisse im Abschnitt 4.3 dargestellt sind.

9 Der erhobene Datensatz wurde zur Anpassung an die Bevölkerungsstruktur gewichtet. 


\subsection{Ergebnisse der Befragung}

Die im Folgenden dargestellten Ergebnisse der Befragung fokussieren sich, bezugnehmend auf die beschriebenen theoretischen Annahmen, auf drei wesentliche Fragestellungen: (1.) selbstberichtete Delinquenz, (2.) Einstellungen zur Legitimität der Polizei und (3.) moralische Einstellungen und Strafverfolgungsrisiko.

\subsubsection{Selbstberichtete Delinquenz}

Die beiden Fragestellungen zur selbstberichteten Delinquenz bezogen sich auf Normkonformität in der Vergangenheit und etwaige Änderungen der Einstellung in der Zukunft. Insgesamt gaben $92 \%$ der befragten Personen an, keine unerlaubten Handlungen vorgenommen zu haben, wobei die Normtreue, wie aus der untenstehenden Tabelle 1 ersichtlich wird, von der Art des Deliktes abhängig war. Die etwaige Begehung einer Straftat in der Zukunft war auf die Fragen c. bis e. beschränkt. Durchschnittlich $95 \%$ hielten es für unwahrscheinlich oder eher unwahrscheinlich zukünftig die genannten Straftaten zu begehen.

Tabelle 1 Selbstberichtete Delinquenz und Einstellung zur Begehung einer Straftat in der Zukunft

\begin{tabular}{llll}
\hline strafbewährte Handlung* & $\begin{array}{l}\text { niemals in den letz- } \\
\text { ten fünf Jahren vorge- } \\
\text { nommene Straftat }\end{array}$ & $\begin{array}{l}\text { unwahrscheinliche oder } \\
\text { eher unwahrscheinliche } \\
\text { Begehung einer Straftat } \\
\text { in der Zukunft }\end{array}$ \\
\hline a $\quad \begin{array}{l}\text { Herunterladen von Musik, Fernsehsendungen } \\
\text { oder Filmen von Internetseiten, die illegal sein } \\
\text { könnten }\end{array}$ & $85,43 \%$ & - \\
b $\quad$ Diebstahl einer DVD aus einem Geschäft & $97,09 \%$ & - \\
c $\quad$ Kauf von möglicherweise gestohlenen Waren & $93,82 \%$ & $96,48 \%$ \\
d $\quad \begin{array}{l}\text { Kauf von geschmuggelten, unverzollten Waren, } \\
\text { z. B. Alkohol oder Zigaretten }\end{array}$ & $89,63 \%$ & $93,60 \%$ \\
e $\quad \begin{array}{l}\text { Beschäftigung einer Person, die keine Arbeitser- } \\
\quad \text { laubnis für Deutschland hat, z. B. als Handwerker } \\
\text { oder für die Kinderbetreuung }\end{array}$ & $95,19 \%$ & $94,09 \%$ \\
\hline
\end{tabular}

* Die Auswahl der unerlaubten Handlungen ergibt sich aus dem Projekt Fiducia. Sie können dem Bereich der Alltagsdelikte zugerechnet werden und sind aufgrund der ähnlichen Strukturen auch auf andere Delikte aus dem Bereich der Alltagsdelikte übertragbar. 


\subsubsection{Moralische Einstellung und Strafverfolgungsrisiko}

Als weitere Faktoren wurden die moralische Einstellung zu den unter 4.2.1 genannten Delikten sowie die Wahrscheinlichkeit ihres Strafverfolgungsrisikos einbezogen.

Insgesamt bewerteten $92 \%$ eine der Verhaltensweisen (a. bis e.) für falsch oder eher falsch, wobei sich die Angaben je nach Deliktsart unterschieden: a: $85 \%$, b: $98 \%$, c: $95 \%$, d: $94 \%$, e: $86 \%$. Eine Bestrafung hielten die Befragten wie folgt für wahrscheinlich oder eher wahrscheinlich: a: $57 \%$, b: $79 \%$, c: $61 \%$, d: $60 \%$, e: $72 \%$.

\subsubsection{Einstellungen zur Legitimität der Polizei}

Die Anerkennung der Legitimität als Prädiktor für strafbares Verhalten ist eine der Kernfragen dieses Beitrags. Da für die meisten Personen die Polizei am ehesten für Recht und „die Macht des Staates“ steht, wurde sie stellvertretend für den Begriff Institutionen ausgewählt. Die Fragestellungen bezogen sich auf die normative Übereinstimmung von polizeilichem Verhalten und eigenen Einstellungen sowie die moralische Verpflichtung, polizeilichen Anweisungen Folge zu leisten.

Bei den normativen Übereinstimmungen stimmten von den Befragten den folgenden Aussagen zu oder voll und ganz zu: (a.) die Polizei trifft Entscheidung, die dem Verständnis der Befragten von richtig oder falsch entsprechen (74\%), (b.) der Polizei kann getraut werden, die richtigen Entscheidungen zu treffen (65\%), (c.) die Polizei hat im Allgemeinen dieselbe Auffassung von richtig und falsch wie der Befragte (61\%). Bei den Fragen nach der moralischen Verpflichtung konnten die Befragten auf einer Skala von o (trifft überhaupt nicht $\mathrm{zu}$ ) bis 10 (trifft voll und ganz zu) antworten. Sie gaben an: ${ }^{10}$ (a.) eine Entscheidung der Polizei als rechtmäßige Behörde zu akzeptieren (7), (b.) eine Entscheidung der Polizei zu akzeptieren, selbst wenn sie mit der Entscheidung nicht einverstanden sind (6), (c.) zu machen, was die Polizei sagt, selbst wenn sie die Gründe nicht verstehen oder nicht damit einverstanden sind (6).

\subsection{Ergebnisse der Befragung}

Um festzustellen, welcher Prädiktor Einfluss auf zukünftiges strafbares Verhalten hat, wurden die erhobenen Daten anhand eines negativen, binomialen Regressionsmodells ausgewertet. Bezüglich der selbstberichteten Delinquenz und Wahrscheinlichkeit zukünftigen delinquenten Verhaltens wurde eine neue Variable geschaffen. Die Prädiktoren sind: die normative Übereinstimmung, die moralische Einstellung, das Risiko einer Bestrafung sowie Alter und Geschlecht. Die Pflicht zu gehorchen entfiel als Prädiktor für Legitimität, da beide Komponenten stark korrelierten und sich die normative Übereinstimmung als der signifikante Prädiktor herausstellte. Aus

10 In den Klammern wird der Mittelwert angegeben. 
Tabelle 2 Vorhersage von selbstberichteter Delinquenz und der Wahrscheinlichkeit ein Delikt zu begehen

\begin{tabular}{llll}
\hline & $\begin{array}{l}\text { normative Über- } \\
\text { einstimmung }\end{array}$ & $\begin{array}{l}\text { Risiko einer } \\
\text { Bestrafung }\end{array}$ & $\begin{array}{l}\text { moralische } \\
\text { Beurteilung }\end{array}$ \\
\hline $\begin{array}{l}\text { selbstberichtete Delinquenz } \\
\begin{array}{l}\text { Wahrscheinlichkeit eines zukünftigen } \\
\text { strafbaren Verhaltens }\end{array}\end{array}$ & neg. Assoziation & pos. Assoziation & neg. Assoziation \\
\hline
\end{tabular}

Tabelle 2 lässt sich entnehmen, dass die Wahrscheinlichkeit selbstberichteter Delinquenz aufgrund der hohen normativen Übereinstimmung zwischen den Werten der Polizei und den eigenen Werten sowie der moralischen Verurteilung von strafbarem Verhalten gering ist. Im Hinblick auf ein zukünftiges strafbares Verhalten ist die moralische Einstellung der stärkste Prädiktor. Hält jemand die Begehung eines Deliktes für falsch, ist es wahrscheinlich, dass er zukünftig davon absieht. Das Strafverfolgungsrisiko und die Legitimität haben keine signifikanten Auswirkungen.

Die Sorge vor einer Bestrafung als schwacher Prädiktor für zukünftiges Verhalten zeigt eine weitere Grenze repressiver Strategien auf. Zudem verursachen sie, wie beschrieben, erhebliche Kosten und scheinen ein Kampf gegen Windmühlen zu sein, da sich die unerlaubten Handlungen ungehindert fortsetzen, ob nun bei der Schwarzarbeit, dem Kauf geschmuggelter Zigaretten oder gefälschter Produkte oder dem Schwarzfahren (vgl. Bundesministerium der Finanzen 2017, S. 10, 12, 18; Statista 2017). Selbst bei vorangekündigten Kontrollen wurden im Juni 2017 in Hamburg noch 3,7 \% der kontrollierten Fahrgäste ohne gültigen Fahrausweis angetroffen. Die Dunkelziffer dürfte um ein Vielfaches höher sein. Die Täter lassen sich von verstärkten Kontrollen selten abhalten, sondern halten das Risiko einer Bestrafung für gering oder ändern ihre Strategien. Anhand von Kontrollmaßnahmen kann nur ein kleiner Teil der Transaktionen, Dienstleistungen oder Fahrten in öffentlichen Verkehrsmitteln überwacht werden. Nur wenige Täter können strafrechtlich verfolgt werden, so dass eine geringe Sorge vor einer Entdeckung und Bestrafung besteht. Die Abnehmer unerlaubt gehandelter Produkte lassen sich, sofern sie sich überhaupt strafbar machen, aufgrund der geringen Entdeckungswahrscheinlichkeit von Kontrollmaßnahmen und verschärften Gesetzen nicht beeindrucken.

Dagegen bieten die moralischen Einstellungen als ein starker Prädiktor für Normkonformität einen Anhaltspunkt für normative Strategien. Bei der Entwicklung der Strategien ist auch einzubeziehen, dass zwar ein Großteil der Befragten die Begehung der genannten Delikte für falsch hält, aber knapp $42 \%$ der Aussage zustimmten, dass man Gesetze missachten könne, wenn niemand Schaden nehme. Im Hinblick auf Alltagsdelikte sollte daher auf die Folgen für Unternehmen, die deutsche Wirtschaft, die Gesundheit sowie die etwaige Unterstützung der organisierten Kriminalität oder 
Kinderarbeit hingewiesen werden. Von der organisierten Kriminalität distanzieren sich Konsumenten illegaler Produkte deutlich und wollen sie nicht unterstützen (vgl. Stead et al. 2013, S. 2216). In einer von PricewaterhouseCoopers ( $\mathrm{PwC}$ ) durchgeführten Studie gaben $66 \%$ der befragten Briten an, dass sie moralische oder ethische Bedenken vom Kauf gefälschter Produkte abhalten würden (vgl. PwC 2013, S. 7). Ein weiterer einzubeziehender Faktor ist die soziale Akzeptanz. Nur $63 \%$ der innerhalb des Projektes Fiducia Befragten gehen davon aus, dass ihre Freunde den illegalen Download missbilligen. Beim Schmuggel von Zigaretten oder Alkohol sind es $73 \%$, bei der Beschäftigung einer Person ohne Arbeitserlaubnis $70 \%$.

\section{$5 \quad$ Schlussfolgerung}

Auch wenn soziale und rechtliche Normen durch den täglichen Umgang eingeübt und in den Alltag des Einzelnen integriert sind, kommt es im Bereich der Alltagsdelikte immer wieder zu abweichendem Verhalten des Einzelnen, das oft sozial akzeptiert und durch verschiedene Neutralisierungstechniken gerechtfertigt wird. Normative kriminalpräventive Maßnahmen sollten folgende Ziele verfolgen: (1.) die Kongruenz moralischer Einstellungen und rechtlicher Regelungen und eine dahingehende Moral- und Werteerziehung, (2.) die Internalisierung von Normen in allen Bereichen, (3.) die Sensibilisierung und Verdeutlichung des geltende Rechts.

\subsection{Sensibilisierung des Rechtsbewusstseins}

Wie festgestellt, sind moralische Einstellungen ein wichtiger Prädiktor für zukünftige Straftaten. Daher ist es wichtig zu vermitteln, was falsch und was richtig ist. Die Vermittlung von Rechtsbewusstsein und Moral kann durch Vorbilder vorgenommen werden, durch Öffentlichkeitskampagnen oder Ethikcodes in Unternehmen. Die Kampagne "Schwarzrauchen - eine miese Nummer" (2008) von Philip Morris informierte die Verbraucher über die Konsequenzen des unerlaubten Zigarettenhandels, wie eine verminderte Qualität der Zigaretten, Steuereinbußen und eine Verbindung zur organisierten Kriminalität (vgl. Philip Morris und Bundesverband des Tabakwaren-Einzelhandels e.V. 2008). British-American Tobacco versucht, mit dem Video „Das ist der Mann“ die Öffentlichkeit zu erreichen. Das Video klärt über etwaige Zusammenhänge des unerlaubten Handels mit Kinderarbeit, Menschenhandel und der organisierten Kriminalität auf, um zu verdeutlichen, dass der unerlaubte Zigarettenhandel keinesfalls eine Straftat ohne Opfer ist. Aber auch internationale Konventionen, die nicht immer rechtliche Verpflichtungen enthalten, können ein erster Schritt sein, um ein Problem zu beschreiben, die Gesellschaft zu sensibilisieren und dem Konsumenten, potentiellen Tätern aber auch Wirtschaftsunternehmen eine moralische Ausrichtung zu bieten (vgl. McDonald und Roberts 1994, S. 59). Informa- 
tionskampagnen sollen aber nicht nur der Sensibilisierung für ein Problem und der Entwicklung eines Unrechtsbewusstseins dienen, sondern auch der Internalisierung von Normen.

\subsection{Unterbrechung der sozialen Akzeptanz von Straftaten}

Die Sensibilisierung über das, was moralisch richtig ist, betrifft diejenigen, die zwar keine Straftaten begehen, aber das abweichende Verhalten ihres sozialen Umfeldes nicht missbilligen. Nach Leary et al. ist die soziale Akzeptanz ein wichtiger Bestandteil menschlichen Verhaltens sowie des Selbstbewusstseins und geeignet, Verhalten zu beeinflussen (vgl. Leary et al. 2001, S. 898). Vor allem denjenigen, die sich für integrierte Mitglieder der Gesellschaft halten, ist die gesellschaftliche Akzeptanz ihres Verhaltens wichtig. Wird das abweichende Verhalten nicht akzeptiert, sondern stigmatisiert, ist davon auszugehen, dass Personen ihre Einstellungen und ihr Verhalten ändern, sodass es schlussendlich zu einer Abnahme von Alltagsdelikten kommt. Ein Beispiel für eine Änderung der sozialen Akzeptanz ist das Nichtrauchen. War es vor wenigen Jahrzehnte noch üblich, dass in öffentlichen Gebäude, Zügen und Gaststätten geraucht wurde, ist es heutzutage allgemeiner Konsens, es nicht zu tun.

\subsection{Kontrollen und Bestrafung}

Wie im Abschnitt 3.1 erwähnt, sind das Verlangen nach günstigen Produkten, finanzielle Vorteile oder die Tolerierung des illegalen Verhaltens durch Strafverfolgungsbehörden treibende Faktoren. Daher kann aus spezial- und generalpräventiven Gründen auch bei den Delikten der Alltagskriminalität nicht grundsätzlich auf Kontrollen und Bestrafung verzichtet werden. Die Kontrollen dienen nicht nur zur Aufdeckung unerlaubten Verhaltens, sondern auch der Sensibilisierung des Unrechtsbewusstseins und des Rechtsempfindens. Der eingangs zitierte Vorschlag, Alltagskriminalität nicht als Straftat, sondern Ordnungswidrigkeit zu verfolgen, reduziert zwar die Kosten für ein Strafverfahren, aber nicht die Delikte und die durch sie verursachten Schäden.

\subsection{Ergebnis}

Abschließend lässt sich feststellen, dass repressive aber auch normative Strategien ihre Grenzen haben. Eine effektive Kriminalprävention kann heute jedoch nicht allein auf repressive Kontrollmechanismen gestützt werden. Um Normkonformität bei Alltagsdelikten zu erreichen, sollte die Förderung interner Mechanismen (moralischer Verpflichtung) sowie des Vertrauens von Menschen in das Rechtssystem und die Legitimität von Normen einbezogen werden. Denn die Täter, gerade im Be- 
reich der Alltagsdelikte, lassen sich nicht nur von rationalen, sondern auch normativen Erwägungen leiten. Das setzt die Kenntnis von Normen voraus verbunden mit der Akzeptanz, dass das verbotene Verhalten moralisch falsch ist. Die Förderung von Normakzeptanz und die Unterbrechung der sozialen Akzeptanz von Normverstößen können wichtige Schritte sein, um Alltagsdelikte zu reduzieren. Im Ergebnis scheint es für eine effektive Kriminalprävention unverzichtbar, die verschiedenen Ansätze miteinander zu verbinden. Auch wenn der Erfolg normativer Strategien, die auf Vertrauen in die Legitimität von Normen gerichtet sind, eine Änderung gesellschaftlicher Einstellungen voraussetzen und keine kurzfristigen Erfolge bringen, zeigt sich ausreichend Potential für ihre Anwendung.

\section{Literatur}

Antonopoulos, G. A. (2008). The Greek Connection(s): The Social Organization of the Cigarette-Smuggling Business in Greece. European Journal of Criminology, 5, 3, 263288.

Becker, G.S. (1968). Crime and punishment: an economic approach. Journal of Political Economy, Vol. 78, 169-217.

Beetham, D. (1991). The Legitimation of Power. Basingstoke: Palgrave Macmillan.

Bottoms, A.E. (2001). Compliance and community penalties. In A.E. Bottoms (Hrsg.), Community penalties change and challenges (S. 87-116). Cullompton: Willan.

Bundesministerium der Finanzen (2017). Der Zoll - Jahresstatistik 2016. www.bundes finanzministerium.de/Content/DE/Downloads/Broschueren_Bestellservice/2017-04o4-zoll-jahresstatistik-2016.pdf?_blob=publicationFile\&v=6. Zugegriffen: o1. Juli 2017.

Bundesverband Musikindustrie (o. D.). Internetpiraterie. www.musikindustrie.de/inter netpiraterie. Zugegriffen: O1. Juli 2017.

Bräuninger, M., \& Stiller, S. (2010). Ökonomische Konsequenzen des Konsums von nicht in Deutschland versteuerten Zigaretten. www.zigarettenverband.de/pos-data/page_ img/Publikationen/Pressemitteilungen/HWWI_Policy_Paper_1-28.pdf. Zugegriffen: o1. Juli 2017.

Eidgenössische Zollverwaltung (2017). Einfuhr in die Schweiz. www.ezv.admin.ch/ezv/ de/home/information-private/reisen-und-einkaufen--freimengen-und-wertfreigren ze/einfuhr-in-die-schweiz.html. Zugegriffen: 01. Juli 2017.

Enste, D. (2017). Schwarzarbeit und Schattenwirtschaft - Argumente und Fakten zur nicht angemeldeten Erwerbstätigkeit in Deutschland und Europa. Köln: Institut der deutschen Wirtschaft.

Falk, A. (2001). Homo Oeconomicus versus Homo Reciprocans: Ansätze für ein neue wirtschaftspolitisches Leitbild. Working Paper No. 79. Universität Zürich: Working Paper Series. 
Glaser, K. (2013). Über legitime Herrschaft - Grundlagen der Legitimitätstheorie. Wiesbaden: Springer VS.

Gewerkschaft der Polizei (2015). Alltagsdelikte schneller und effektiver verfolgen. www. gdp.de/gdp/gdpnrw.nsf/id/de_alltagsdelikte-schneller-und-effektiver-verfolgen. Zugegriffen: O1. Juli 2017.

Hansen, K. (2012). Zigarettenschmuggel: Berliner Alltäglichkeit. www.tagesspiegel.de/ber lin/illegaler-verkauf-selbst-wenn-wir-10o-zoellner-mehr-haetten-wuerden-wir-dasproblem-nicht-in-den-griff-bekommen/6060916-2.html. Zugegriffen: 01. Juli 2017.

Hilgendorf, E. (2001). Recht und Moral. Aufklärung und Kritik, I/2001, 72-90.

Jackson, J., Bradford, B., Hough, M., Myhil, A., Quinton, P., \& Tyler, T. R. (2012). Why people obey the law? Legitimacy and the Influence of Legal Institutions. British Journal of Criminology, 52, 1051-1071.

Jiang, L., \& Cova, V. (2012). Love for Luxury, Preference for Counterfeits - A Qualitative Study in Counterfeit Luxury Consumption in China. International Journal of Marketing Studies, 4, 6, (S. 1-9).

Karstedt, S., \& Farrall, S. (2006). The Moral Economy of Everyday Crime: Markets, Consumer and Citizens. British Journal of Criminology, 46, 1011-1036.

Leary, M.R., Cottrell, C.A., \& Phillips, M. (2001). Deconfounding the effects of dominance and social acceptance on self-esteem. Journal of Personality and Social Psychology, 81, 5, 898-909.

Lin-Hi, N. (o. D.). Homo Oeconomicus. http://wirtschaftslexikon.gabler.de/Archiv/8004/ homo-oeconomicus-v12.html. Zugegriffen: 01. Juli 2017.

Lind, E. A., \& Tyler, T. R. (1988). The social psychology of procedural justice. New York: Plenum.

Mazar, N., \& Ariely, D. (2006). Dishonesty in Everyday Life and Its Policy Implications. Journal of Public Policy and Marketing, Vol. 25, No. 1, 117-126.

McDonald, G., \& Roberts, C. (1994). Product Piracy: The Problem that will not go away. Journal of Product \& Brand Management, 3, 4, 55-65.

Niemand, T. (2014). Produktpiraterie: Eine Integrative Analyse der Einflussgrößen, Moderatoren und Mediatoren des Erwerbs von Fälschungen und Raubkopien. Wiesbaden: Springer Gabler.

Philip Morris, \& Bundesverband des Tabakwaren-Einzelhandels e. V. (2008). Schwarzrauchen - eine miese Nummer. www.tabakwelt.de/cms/branchenthemen/PDFs/Schwarz rauchen_Factsheet.pdf. Zugegriffen: 01. Juli 2017.

PwC (2013). Counterfeit goods in the UK - Consumer survey October 2013. www.pwc. co.uk/en_UK/uk/assets/pdf/anti-counterfeiting-consumer-survey-october-2013.pdf. Zugegriffen: o1. Juli 2017.

Reich, A. (2015). Abschied vom Homo Oeconomicus. www.zeit.de/kultur/2015-08/wirt schaftspolitik-tania-singer-resource-projekt-1onach8. Zugegriffen: 01. Juli 2017.

Singer, T. (2015). How to build a caring economy. www.weforum.org/agenda/2015/o1/ how-to-build-a-caring-economy. Zugegriffen: o1. Juli 2017. 
Statista (2017). Umfrage zum Fahren ohne gültigen Fahrschein im ÖPNV in Deutschland nach Alter im Jahr 2017. http://statista-research.com. Zugegriffen: 01. Juli 2017.

Stead, M., Jones, L., Docherty, G., Gough, B., Antoniak, M., \& McNeill, A. (2013). ,Noone actually goes to a shop and buys them do they?': attitudes and behaviours regarding illicit tobacco in a multiply disadvantaged community in England. Addiction, 108, 12, 2212-2219.

Sykes, G. M., \& Matza, D. (1957). Techniques of Neutralization: A Theory of Delinquency. American Sociological Review, 22, 6, 664-670.

Tajfal, H., \& Turner, J. C. (1986). The Social Identity Theory of Intergroup Behaviour. In S. Worchel, \& W. G. Austin (Hrsg), Psychology of Intergroup Relations (S. 7-24). Chicago: Nelson-Hall.

Thompson, E. P. (1971). The Moral Economy of the English Crowd in the Eighteenth Century. Past and Present, Vol. 50, 76-136.

Tyler, T. R. (1990). Why people obey the law. Yale University Press: New Haven.

Tyler, T.R. (2003). Procedural Justice, Legitimacy, and the Effective Rule of Law. Crime and Justice, 30, 283-357.

Verband Deutscher Verkehrsunternehmen (2015). Bundesrat stimmt zu - Schwarzfahren kostet ab Juli 6o Euro. www.vdv.de/150508-pm-ebe-beschluss-im-bundesrat.pdfx?for ced=true. Zugegriffen: o1. Juli 2017.

Vollmuth, J. (2009). Studie zeigt Motive für Fälschungskauf von Elektronikprodukten im Internet auf. www.elektronikpraxis.vogel.de/themen/elektronikmanagement/recht produkthaftung/articles/189073/index2.html. Zugegriffen: 01. Juli 2017.

Wagner, M. (2010): Die moralische Ökonomie des Schmuggels. In M. Wagner, \& W. Łukowski (Hrsg.), Alltag im Grenzland (S. 73-89). Wiesbaden: Springer VS.

Wagner, M. (2011). Die Schmugglergesellschaft - informelle Ökonomien an der Ostgrenze der Europäischen Union. Bielefeld: transcript.

Wilcox, K., Kim, H. M., \& Sen, S. (2009): Why Do Consumers Buy Counterfeit Luxury Brands? Journal of Marketing Research, 46, 2, 247-259.

Zoll (2017a). Genussmittel: Rückkehr aus einem anderen EU-Mitgliedsstaat. www.zoll.de/ DE/Privatpersonen/Reisen/Reisen-innerhalb-der-EU/Steuern/Genussmittel/genuss mittel_node.html\#doc18312bodyText1. Zugegriffen: o1. Juli 2017.

Zoll (2017b). Reisefreimengen. www.zoll.de/DE/Privatpersonen/Reisen/Rueckkehr-auseinem-Nicht-EU-Staat/Zoll-und-Steuern/Reisefreimengen/reisefreimengen_node. html. Zugegriffen: 01. Juli 2017. 